\title{
PRAGMÁTICA Y CORPUS DE LENGUAJE DISFUNCIONAL. UN CAMINO DE ASUNCIONES Y PARADOJAS*
}

\author{
MILAGROS FERNÁNDEZ PÉREZ \\ Universidade de Santiago de Compostela
}

"La linguistique s'intéresse au langage sous tous aspects -au langage en acte, au langage en évolution, au langage à l'état naissant, au langage en dissolution"

(Jakobson, 1956: 43)

\section{RESUMEN}

En este trabajo se subrayan dimensiones de concurrencia entre aspectos pragmáticos y cultivo de lingüística de corpus. Se destaca asimismo la necesidad de definir con precisión qué elementos de praxis son imprescindibles para tratar muestras de producciones reales en un repertorio de datos. Se insiste sobre todo en qué características interactivas deben marcarse en inventarios de lenguaje disfuncional. Las peculiaridades del habla mermada incrementan las dificultades de determinación. En el trabajo se hace un trazado de las grandes líneas que han orientado la inclusión de principios y funciones pragmáticas en corpus de habla disfuncional (\$3), y con objeto de aportar criterios homogéneos sólidos al respecto. No sin antes examinar las rutas de conexión entre Pragmática y Lingüística de corpus $(\$ 1)$ y las bases de elaboración de los inventarios tildados de corpus pragmáticos $(\$ 2)$.

PALABRAS ClaVE: pragmática, lingüistica de corpus, corpus pragmáticos, lenguaje disfuncional, corpus de lenguaje clínico, perspectiva Pragmática, pragmática clínica.

\section{ABSTRACT}

This work highlights some aspects about the coinciding dimensions in pragmatic aspects and corpus Linguistics. It also stresses the need for defining accurately which elements within the praxis are unavoidable in order to work with a sample of real productions taken from a database. The emphasis is especially put on the interactive characteristics that should be marked in inventories of dysfunctional language, as the peculiarities of diminished language increase the difficulty when it comes to determine them. This work traces the pathways that

* Este trabajo se inscribe en el proyecto Procesos fónicos y emergencia de sonidos idiomáticos (FFI2013-41851-P. Ministerio de Economía y Competitividad). 
have oriented the inclusion of principles and pragmatic functions in dysfunctional language corpora $(\$ 3)$, and pursues the main goal of providing with homogeneous and solid criteria to fulfill this task. The connections between Pragmatics and Corpus Linguistics $(\$ 1)$, and the basis for elaborating inventories named pragmatic corpora $(\$ 2)$ have been previously examined.

KEYWORDS: pragmatics, corpus linguistics, pragmatic corpora, dysfunctional language, clinical language corpora, pragmatic perspective, clinical pragmatics.

\section{Presentación}

Moverse en la esfera de la Lingüística de Corpus parece comportar, por lógica, el trabajo con datos procedentes de muestras comunicativas en coordenadas naturales de intercambio. De modo que se asume -quizás con excesiva confianza y convicción- que las características de praxis figuran como parámetros habituales en la composición y etiquetado de la mayor parte de los repertorios. El panorama real, sin embargo, se aleja en cierta medida de esta imagen. Al tiempo que son escasos los inventarios que integran dimensiones pragmáticas, hay también notable diversidad en criterios de anotación en cuanto a lo que son valores de práctica verbal. Rühleman y Aijmer (2015) aluden a lo que podría dibujarse como concurrencia y acaso disputa de intereses entre Pragmática y Lingüística de corpus. En una línea similar, Romero-Trillo (2008) ha señalado la necesidad de apuntalar cometidos y estilos metodológicos entre las dos áreas cuando se abordan muestras de producciones y se analizan interacciones lingüísticas.

La relativa imprecisión general entre pragmática y corpus, y también la ausencia de pautas bien definidas cuando se trata de componer repertorios de datos etiquetados en sus valores interactivos, incrementan su tono en el caso de inventarios de lenguaje disfuncional. A los inconvenientes mencionados se añaden otros derivados de las peculiaridades consustanciales al lenguaje mermado. En este trabajo se hace un trazado de las grandes líneas que han orientado la inclusión de principios y funciones pragmáticas en corpus de habla disfuncional (\$3) con objeto de aportar criterios homogéneos sólidos al respecto. No sin antes examinar las rutas de conexión entre Pragmática y Lingüística de corpus (\$1) y las bases de elaboración de los inventarios tildados de corpus pragmáticos (\$2).

\section{LINGÜÍSTICA DE CORPUS Y PRAGMÁTICA. INTERFAZ, RELACIONES Y PRISMAS ENTRE DOMINIOS}

Las dificultades para diferenciar las dos áreas, así como la indeterminación e incluso la borrosidad cuando se trata de atribuirles encargos exclu- 
sivos y diferenciados, surgen en tradiciones que distinguen estrictamente componentes. En la lingüística anglosajona, ligada a la tradición británica y con visión global que respeta la simultaneidad de la realidad comunicativa y sin necesidad de marcar fronteras entre dimensiones de la lengua, es habitual asumir la presencia de aspectos pragmáticos en la elaboración de corpus $^{1}$. Es por ello que el llamado "prisma de praxis" resulta inherente en estudios sobre repertorios como los de Hunston (2002, 2009) o Biber (2006, 2010, 2011). Es en tales coordenadas en donde comúnmente se confeccionan corpus con etiquetas pragmáticas ligadas al análisis de las muestras compiladas.

En general, los inconvenientes en la delimitación y la polémica sobre las competencias, la esfera de operatividad y las confluencias de cada una de las áreas se deben a visiones excesivamente centradas en cometidos y análisis privativos. Romero-Trillo (2008), después de un examen riguroso sobre concepciones de la Lingüística de corpus -y que van desde su identificación mecanicista y cuantitativa (visión destacada en Gries, 2010) hasta la versión de perspectiva metodológica en lingüística y patente en inventarios anotados (propia de Chafe, 1992; o de Teubert, 2005, y Teubert y Krishnamurthy, 2007), sin olvidar aquella otra que conjuga los datos en su probabilidad teórica (Halliday, 1992)-, considera que los objetivos instrumentales y de procedimiento que la asimilan a lo puramente cuantitativo no se compadecen con las miras analíticas de los repertorios, siempre necesitados de complemento explicativo e interpretativo en las categorías y en los aspectos descriptivos de la lengua. De algún modo, Lingüística de corpus y Pragmática son disciplinas abocadas a lograr entendimiento mutuo, tal y como reza en su título, "A mutualistic entente", el trabajo de Romero-Trillo. Con sus palabras:

Corpus linguistics and pragmatics are two versions of the same phenomenon: the mechanics -the subject-matter- (corpus studies), and its interpretation and explanation (pragmatics) (Romero-Trillo, 2008: 5).

En una línea similar de contundencia, Rühlemann y Aijmer (2015) enuncian argumentos de la mano de exhaustivos exámenes sobre los modi operandi en las dos esferas. De entrada, reconocen una ruta propia de cada área en la aproximación a las categorías. En Lingüística de corpus es el prisma en vertical lo que conduce a las unidades relevantes: las llamadas ocurrencias de un segmento en distintas distribuciones son herramienta

\footnotetext{
${ }^{1}$ El reciente trabajo de Battaner (2014) instruye con claridad meridiana sobre tal concepción y también sobre la presencia de conceptos como contexto de situación, acción comunicativa, colocación, coligación, en la lingüística británica de Firth (1890-1960), antecedente y reflejo de enfoques actuales en Lingüística de corpus.
} 
clave en el trabajo con corpus. No en vano el cometido es hallar patrones de combinatoria mediante el estudio de frecuencias de ocurrencias ${ }^{2}$. Por contra, en Pragmática la pertinencia del contexto se constituye en estelar, así que no es posible obviar el enfoque con rumbo en horizontal: los valores significativos se definen en cada contexto. En su opinión:

The focus is not on the number of occurrences but on the functional behaviour observable in the texts of the phenomena under examination. Given the dependence on context, pragmatic research has methodologically relied on the analysis of small numbers of texts where careful "horizontal" reading is manageable (Rühlemann y Aijmer, 2015: 3).

El desarrollo metodológico tanto de la Pragmática como de la Lingüística de corpus conduce, por natural, a vías de confluencia que exigen conjugar los dos abordajes. Una concepción rica de la Lingüística de corpus no se ciñe simplemente a ocurrencias, sino que da cabida a colocaciones y a concordancias. Lo que se ha dado en llamar anotación KWIC, Key Word in Context, ha servido para constatar que el significado no se halla en palabras individuales, sino en "units of meaning" (Thompson y Hunston, 2006: 11), de modo que se requiere comprobar las distribuciones (concordancias) para derivar las colocaciones y los patrones relevantes de combinatoria contextual. Una palabra como "corpus" figura combinada (key word in context) en el BNC (British National Corpus) con palabras como "habeas"/"british"/"christi"/ "callosum". La palabra "british" tiene un total de 35.431 ocurrencias, mientras que para "habeas" son 49, para "callosum" 17 y para "christi" 82. A pesar de tales frecuencias, el patrón relevante consecuencia de las colocaciones es el de "corpus christi", ya que son 60 las colocaciones, frente a las 50 de "british corpus”. Según Rühlemann y Aijmer (2015: 9):

the strength expressed in the log-likelihood value that binds "Christi" to "corpus" is much greater than the bond between "british" and "corpus".

Por otra parte, la elaboración de repertorios con datos pragmáticos requiere tomar en cuenta las funciones y etiquetarlas según se hallen las formas ubicadas en los contextos. De manera que las ocurrencias en lo que importa en pragmática están sujetas al análisis por colocación. De hecho, las clasificaciones formales previas no se ajustan a los valores de función contextual: palabras como "bien", "perdón”, "gracias" no son susceptibles de análisis 'de la forma a la función', sino que exigen estudio de sus valo-

\footnotetext{
${ }^{2}$ Berber Sardinha (2004: 40 ss.) señala que la ocurrencia de unidades ponderada en términos de frecuencia sirve para reconocer patrones sistemáticos en la investigación en corpus. Destacar la regularidad es uno de los objetivos del trabajo en corpus, según Thompson y Hunston (2006: 9), y para ello hay que examinar las frecuencias de las colocaciones.
} 
res en contexto, de sus concordancias. Lo cual es propio en fenómenos de corte pragmático, y que constata por tanto la urgencia de anotar tales funciones en los repertorios ${ }^{3}$.

Pragmática y Lingüística de corpus confluyen con intersecciones en su refinamiento y detalle analítico en la medida en que se incrementan las convenciones y los automatismos de etiquetado de propiedades comunicativas. Su camino de integración es patente en aquellos inventarios con codificación ya notable en valores pragmáticos, por lo que la investigación en corpus pragmáticos no equivale a la simple suma de trabajo en corpus más descripción de valores comunicativos, sino que se producen cambios y adaptaciones que redefinen los prismas y los métodos. Con palabras de Rühlemann y Aijmer (2015: 12):

So corpus-pragmatic research is more than just pragmatic research and it is more than just corpus-linguistic analysis in that it integrates the horizontal (qualitative) methodology typical of pragmatics with the vertical (quantitative) methodology predominant in corpus linguistics.

Así, el Narrative Corpus (NC), que se ha configurado sobre el subcorpus conversacional del British National Corpus $(B N C)$, se ha anotado dando cabida a "voces" narrativas (directa/indirecta) con papeles distintos asignados al narrador y con cambios en los actos de habla, hasta el extremo de que:

the analysis started off by means of horizontal reading (identifying speech acts) while the provision of further corpus tools to be subsets added a vertical dimension to the analysis: speech acts could be categorized not only in terms of their type but also in terms of their associated contextual factors (Rühlemann y Aijmer, 2015: 11).

Sin duda, la orientación hacia muestras verbales reales propia de la Lingüística de corpus subraya el protagonismo de la observación por encima de los experimentos o de la intuición del lingüista como hablante con saber tácito. El trabajo de Chafe (1992) resulta crucial para acotar la precisión metodológica de la Lingüística de corpus más allá de técnicas estadísticas, su contribución destaca el papel de la observación natural no-condicionada como modo operativo central en el ámbito, como refleja el esquema:

\footnotetext{
${ }^{3}$ Rühlemann y Aijmer (2015: 10) se refieren a la necesidad de 'normalizar' las anotaciones de valores funcionales en contexto para eludir confusiones y ahuyentar análisis superficiales:

"One way to achieve both high precision and optimal recall when analysing the many pragmatic phenomena characterized by form-function mismatch is by adding annotation targeted at the phenomena one whishes to study".
} 
Figura 1. Cuatro tipos de técnicas de observación

\begin{tabular}{|cll|}
\hline & behavioral & \multicolumn{1}{c|}{ introspective } \\
artificial & $\begin{array}{l}\text { experiments, } \\
\text { elicitation }\end{array}$ & $\begin{array}{l}\text { semantics, judgments regarding } \\
\text { invented language }\end{array}$ \\
& $\begin{array}{l}\text { corpora, } \\
\text { ethnography }\end{array}$ & daydreaming \\
\hline
\end{tabular}

(Chafe, 1992: 66. Figure 1) ${ }^{4}$

En este sentido, el trabajo en Lingüística de corpus traspasa lo cuantitativo y requiere perspectivas sobre valores comunicativos que canalicen la organización de las muestras. La gramática probabilística que defendía Halliday (1992) descansa precisamente en datos que, aunque cuantificados, están fuertemente codificados en propiedades lingüísticas. En su planteamiento de gramática sistémica defiende que:

the frequency in the corpus is the instantiation (note, not realization) of probability in the grammar $[\ldots]$ there is language as a system, an abstract potential, and there are spoken and written texts, which are instances of language in use. But the "system" and the "instance" are not two distinct phenomena. There is only one phenomenon here, the phenomenon of language (Halliday, 1992: 66).

El prisma comunicativo, o lo que se conoce como perspectiva pragmática (McTear y Conti-Ramsden, 1989; Smith y Leinonen, 1992), es indisociable de tareas de confección de corpus. La recolección de muestras, el recurso a parámetros para organizarlas en el depósito, las variables generales para definir la sistemática de datos, son en cualquier caso ingredientes imprescindibles en tareas corpus-driven (Tognini-Bonelli, 2001). Así que la definición de coordenadas macrocomunicativas es habitual en la elaboración

${ }^{4}$ En esta misma línea de definición de rumbo metodológico y de revalorización de las técnicas de observación, Gilquin y Gries (2009) proponen una escala completa de procedimientos para canalizar la obtención de datos lingüísticos en condiciones de naturalidad. Los corpus que contienen muestras de habla o compilaciones de textos escritos figuran en los lugares superiores de la jerarquía, siendo los niveles inferiores los asignados a las vías experimentales (siempre condicionadas). 
de corpus, sobre todo si se trata de inventarios confeccionados en la tradición anglosajona (como el LSWE, Longman Spoken and Written English, a cargo de Biber et al., 1999, o el IDR, Discourse of Interdisciplinary Research, $<$ http://www.birmingham.ac.uk/schools/edacs/departments/english language/research/projects/investigating-interdisciplinary-researchdiscourse.aspx>, bajo la dirección de Thompson y Hunston en la University of Birmingham desde 2005). Son menos abundantes los corpus especializados construidos con diseño inicial de anotación pragmática en microanálisis, si bien, como ya se ha señalado, son factibles codificaciones que implementen paulatinamente el etiquetado en inventarios generales y de referencia. Han sido las investigaciones corpus-oriented las que han promovido tales codificaciones específicas, con el diseño conveniente de marcas relevantes según las muestras y en consonancia con los trastornos considerados.

\section{CORPUS PRAgmáticos. CieRTAS Dificultades y ALgUNOS LOGROS}

En sentido estricto, el interés privativo por los repertorios pragmáticos es muy reciente. En 2004 la revista Journal of Pragmatics dedicó un monográfico a la esfera de los corpus con dimensiones de praxis. Asimismo, en 2007 el Congreso de IPrA versó sobre 'Pragmatics, corpora and computational linguistics', y en 2008 el congreso ICAME convocó la temática 'Corpora: Pragmatics and Discourse'. Posteriormente, y sobre todo en los últimos años, se publican de modo regular compendios sobre cómo se interseccionan los valores pragmáticos con los métodos de la Lingüística de corpus. Entre otros, destacan la serie Yearbook of Corpus Linguistics and Pragmatics bajo la batuta de Romero-Trillo (2014, 2015), Felder et al. (2011) y Jucker y Taavitsainen (2014). En cualquier caso, ha comenzado a borrarse la idea de que son campos "parallel bit often mutually exclusive" (RomeroTrillo, 2008: 2) y se ha iniciado la senda del "entente mutuo".

En sentido amplio, tomando el prisma comunicativo como consustancial en los intercambios verbales reales, la perspectiva pragmática siempre ha formado parte de los procedimientos de observación de las producciones. Los marcos de interacción están presentes en la recogida de muestras y en su posterior análisis. De un modo o de otro, se han tomado en cuenta parámetros contextuales y hormas dialógicas. No sólo los corpus que recogen variedades y registros, o aquellos otros que depositan conversaciones, o los inventarios de un determinado tipo de habla o lecto, sino que en general toda compilación de habla oral o de textos escritos se determina en sus valores comunicativos por el origen de la relevancia lingüística de sus intercambios. Un repertorio de habla juvenil (como, por ejemplo, el COLT, 
The Bergen Corpus of London Teenage Language < http://clu.uni.no/icame/ colt>, también el COLA, Corpus Oral de Lenguaje Adolescente, <http://www. colam.org/transkripsjoner-espannol.html>) sustenta la importancia de sus datos en que son comunicativos, simbólicos e interactivos entre usuarios (hablantes-oyentes jóvenes) en marcos precisos de intercambio espontáneo y distendido ${ }^{5}$.

Los corpus del inglés académico (como son MICASE, Michigan Corpus of Academic Spoken English, <http://quod.lib.umich.edu/cgi/c/corpus/cor pus?c=micase;page=simple $>$, y BASE, British Academic Spoken English Corpus, $<$ http://www.reading.ac.uk/AcaDepts/ll/base_corpus/>) se han elaborado sobre la base de atribuir pertinencia comunicativa especial a un registro formal que funciona en un marco de interacción definido. Por tratarse de un repertorio de habla singular, un corpus especializado de habla de afásicos requiere en mayor medida la definición previa de parámetros de interacción, de rasgos de participación de hablantes y oyentes, y de elementos no-verbales claves en la eficiencia de los intercambios. El prisma pragmático resulta, pues, inevitable en la compilación de muestras verbales naturales. Entendiendo pragmático en el sentido de "language use and language users in interaction" (Bublitz y Norrick, 2011: 4), y con los indicadores que ya señalaba Ochs (1996: 410): en la situación comunicativa tienen funciones clave los rasgos que identifican a los hablantes, las relaciones con los oyentes, el tipo de actividad y las escalas de apreciación y evaluación que se establezcan en las conversaciones.

Si bien en una primera etapa de desarrollo de la Lingüística de corpus han proliferado inventarios planos sin apenas anotaciones, o tan solo codificados en propiedades fónicas, gramaticales y léxicas, en la actualidad es ya notable la labor de diseño de fuentes de datos especializadas como corpus pragmáticos, así como la continuidad en procesos de etiquetado de tales propiedades en grandes repertorios. Incluso hay ya disponibles técnicas automatizadas y semiautomatizadas para la codificación de algunas características, como es el sistema DART (Dialogue Annotation and Research Tool) de M. Weisser $(2010,2015)$, que se acomoda a los planteamientos básicos del DAMSL (Dialogue Act Markup in Several Layers, Allen y Core, 1997), y a los procedimientos y principios contenidos en el COCONUT Corpus de la University of Pittsburgh (<http://www.pitt.edu/ coconut/coconut-cor pus.html $>$ ), el MONROE Corpus de la University of Rochester (<http://www. cs.rochester.edu/research/speech/monroe>), o el Switchboard Corpus (<http://catalog.ldc.upenn.edu/LDC97S62>).

\footnotetext{
${ }^{5}$ En la presentación del COLA, Jørgensen y Eguía (2014: 6) señalan que "debido a las características particulares de la adolescencia, el análisis de su lenguaje debe partir de su interacción, que es fundamentalmente oral, espontánea y tiene lugar en situaciones distendidas”.
} 
Las razones ya señaladas de concepción integral de la lengua, propias de la tradición lingüística británica, explican la confección de corpus definidos en parámetros comunicativos y contextuales. Y, sin duda, están en el origen del diseño de programas automatizados de concordancias que conducen a las llamadas colocaciones: no basta con etiquetar clases de palabras, no es suficiente con cuantificar las frecuencias de ocurrencia, es imprescindible -como se ha avanzado en $\$ 1$ - reconocer los contextos, hay que incluir el etiquetado KWIC (Key Word in Context) para refinar los valores funcionales precisos. Con palabras de Tognini-Bonelli (2012: 19), solo comprobando "the repeated patterns present in the context of the node" se pueden relativizar las ocurrencias y convertirlas en colocaciones con valor. La estampa pragmática es, pues, patente.

Las investigaciones corpus-oriented sobre aspectos de praxis han sido el acicate para especificar el etiquetado. Una primera fase de proyección pragmática en el ámbito de inventarios ya confeccionados se opera sobre grandes repertorios, que se acometen con objeto de determinar subcorpus (conversacionales, narrativos, evaluativos, dialógicos) y anotarlos en funciones pragmáticas. Sucede con el BNC (British National Corpus) que se ha tomado como fuente para codificar elementos de evaluación y apreciación (Timmis, 2015), o para subrayar marcadores del discurso (Aijmer, 2002, 2015). El cometido es el de progresivamente 'normalizar' la presencia de los valores pragmáticos imprescindibles en muchos casos para reconocer la riqueza de las formas lingüísticas y para solventar confusiones y mezclas entre forma y función. Este motivo a su vez explica las dificultades para automatizar el etiquetado. Con palabras de Rühlemann y Aijmer (2015: 11):

The reason why pragmatic annotation is not yet widely used is simple: the form-function mismatch of most pragmatic phenomena means that automatic assignment of tags will often lack precision, and manual implementation of tags (which is time-and resource-intensive) is unavoidable.

A pesar de lo exigente de las anotaciones, se dispone ya de corpus especializados -de tamaño aceptable- que están codificados en algunas dimensiones y características pragmáticas. Es el caso de 'tipos de actos de habla' y de 'marcadores del discurso' en el SPICE-Ireland (Kallen y Kirk, 2012), de 'quotation' y 'papel de participación' en Narrative Corpus (subcorpus del $B N C$ ) (Rühlemann y O’Donnell, 2012), o de 'politeness' (Danescu-Niculesco-Mizil et al., 2013), entre otros. Las propuestas de marcas y su implementación se discuten en grupos y redes orientados a la homogeneización, como son la plataforma para unificar los marcadores del discurso (Aijmer, 2015), el Linguistic Data Consortium (Weisser, 2015), o el consenso en el DAMSL (Dialogue Act Markup in Several Layers, Allen y Core, 1997). 
3. REPERTORIOS DE HABLA DISFUNCIONAL. ¿QUÉ FUNDAMENTOS COMUNICATIVOS? ¿QUÉ CODIFICACIÓN DE CARACTERÍSTICAS PRAGMÁTICAS?

Si bien algunas de las dificultades mencionadas para los corpus de habla esperable siguen vigentes en el caso de inventarios de habla disfuncional -como la heterogeneidad en el etiquetado y en los sistemas de marcas-, hay sin embargo ciertas ventajas que, por tratarse de habla peculiar, disfrutan los repertorios que recogen muestras de trastornos comunicativos. De algún modo, y para dar carta de naturaleza y relevancia a los datos, las muestras compiladas han de tomarse en su eficacia interactiva antes que en su normalidad prevista, así que los corpus de habla disfuncional se sustentan en criterios de eficiencia pragmática antes que en pautas y reglas de gramática. En línea con el prisma pragmático inherente a la confección de todo repertorio de muestras de trastornos verbales, están asimismo las marcas de interacción y praxis que son habituales en estos inventarios, en muchos casos con predominio incluso sobre características gramaticales, fónicas o léxicas.

\subsection{Lenguaje peculiar e interacción. La eficacia comunicativa como criterio de relevancia}

La Lingüística mayormente cultivada en la tradición europea de corte estructural ha insistido en el modelo de langue como 'sistema' que, por otra parte, tiene como trasfondo el patrón de lengua escrita. De modo que la parole apenas se ha contemplado en sus dimensiones orales de discurso, conversación e interacción. Salvo excepciones, como es el caso de Jakobson y algunas propuestas de la Escuela de Praga, los marcos teóricos en la Lingüística europea continental proporcionan moldes y técnicas descriptivos acomodados a las unidades y a los niveles del sistema. La oralidad, las producciones de habla en marcos discursivos, conversacionales, interactivos, sólo han ido tomando depósito a la par del desarrollo de la Pragmática y conforme se han ido instaurando principios de registro de datos en Lingüística. De alguna manera, ha sido la Lingüística de corpus la que ha conferido relevancia a las muestras verbales orales espóntaneas y en coordenadas de producción natural. El interés por el habla disfuncional en su valor propio es deudor sin duda del giro lingüístico hacia la exploración comunicativa de los usos verbales.

En el plano de la oralidad no cabe pretender unidades como las que funcionan en el sistema estructurado de la langue. Aún más, en coordenadas de habla disfuncional, aunque comunicativa, no es posible sustraerse a la presencia de tácticas interactivas entreveradas con rasgos idiosincráti- 
cos de las emisiones. El habla disfuncional interesa porque comunica a pesar de sus singularidades, está cargada de eficacia porque no pierde valor de interacción. El peso comunicativo de las producciones radica en su fuerza de intercambio. De ahí el papel estelar del prisma de praxis, tal y como subrayan Smith y Leinonen (1992: 143):

Unlike theoretical linguistics, the practical, clinical domain of speech pathology cannot afford to divorce language from communication or ignore the interrelatedness of linguistic levels in communication.

El enfoque comunicativo resulta, pues, obligado cuando se opera con muestras de habla espontánea. El prisma pragmático hace posible observar elementos significativos por su efectividad en el intercambio verbal, y sin necesidad de discriminar su carácter fónico, sintáctico o semántico. De hecho, los usos lingüísticos recogidos de las prácticas interaccionales requieren dicha perspectiva para ser contemplados en sus posibilidades de eficacia o para detectar en ellos inconvenientes y limitaciones. Entre los argumentos que dan relieve a la perspectiva pragmática hay que subrayar los siguientes. En primer lugar, se constata que la interacción no sólo, o no siempre, es únicamente lingüística, sino que hay señales no verbales cargadas de significados. En segundo lugar, no cabe desentenderse de la patente interrelación de las producciones lingüísticas con factores de contexto, motivación, interlocutores, temas, y que se visibiliza en las múltiples estrategias creativas o imitativas de los pacientes y que pueden ser claves de activación de su potencial comunicativo. En tercer lugar, la evidencia de que los intercambios verbales no se dan desnudos sino arropados por el marco de interacción que los define en sus funciones comunicativas, manifiesta una vez más la prevalencia del prisma pragmático. Finalmente, y como dimensión sobresaliente, el protagonismo que detenta el receptor para dar valor a las emisiones por su eficacia, exige contemplar las producciones en coordenadas dialógicas y más allá del hablante en exclusiva.

Las singularidades del habla disfuncional no pueden calificarse, pues, de errores, su valor no se decide por comparación con lo esperable. Se trata de estrategias que conviene estudiar para reconstruir el rompecabezas y hallar el código especial, tal y como señala Crystal (1980: 63):

No existe modo de saber por adelantado si el sistema que vamos a encontrar es un reflejo del sistema presente en la comunidad a la que el paciente pertenece. A veces es evidente que el paciente está utilizando una versión reducida del lenguaje que oye a su alrededor. En muchos casos, sin embargo, el paciente "está a lo suyo": dado que su incapacidad le imposibilita aprender o recordar las reglas de la lengua, ha terminado inventando las suyas propias. La tarea del patólogo es determinar cuáles son esas 
reglas $[\ldots]$ Es un poco como estar haciendo un rompecabezas que puede encajar de muchos modos diferentes para dar lugar a diferentes modelos.

Por otra parte, y desde la naturaleza dinámica que conviene al estudio de usos verbales, las descripciones deben reflejar el carácter evolutivo y cambiante de las emisiones. Así que los procedimientos analíticos han de dar cabida prioritaria a los procesos intervinientes en las producciones reales. En lugar de categorías como sonido bilabial/sordo/fricativo, el interés está en observar procesos fónicos como frontalización/omisión/sustitución. Antes que funciones y relaciones sintácticas como sujeto/coordinación/complemento, lo central deben ser procesos como predicación/concordancia/combinatoria. En lugar de masculino/plural/pasado, lo importante son procesos de lexicalización/simplificación/perífrasis. En coordenadas de praxis toman pertinencia todas aquellas dimensiones que promueven y dan soporte al valor comunicativo e interactivo de las emisiones: intercambios con funciones interpersonales, ideativas y textuales (cfr. Fernández López y Prego Vázquez, 2014). La recomposición del puzle está regida por la eficiencia de las producciones del hablante. El objetivo no radica en hallar unidades delimitadas definidas en el sistema y caracterizadas en términos opositivos, sino más bien en disponer de factores y elementos sobre los que se edifica el discurso. La aproximación es 'bottom-up' y la corriente metodológica idónea para acometer la emergencia de procesos es la que se conoce con el nombre de Gramática de construcciones, enfoque teórico que sin duda facilita la aproximación a las emisiones también disfuncionales ${ }^{6}$.

En cualquier caso, el valor de la interacción en Lingüística clínica se ha visto reforzado en términos generales por la revisión de ciertos trastornos antes considerados sin impacto verbal importante. El denominado síndrome de Williams era, tiempo atrás, paradigma de ilustración de la concepción modular del lenguaje: los pacientes Williams presentan trastornos cognitivos (en otros módulos), pero no se constatan limitaciones en el módulo del lenguaje (= gramática). Sin embargo, las disfunciones pragmáticas que se plasman en las coordenadas verbales son ciertamente llamativas en las personas con este síndrome, quienes no respetan turnos, no interpretan el lenguaje figurado, no manejan distintos hilos de conversación. En una línea similar, para abordar las peculiaridades de ejecución lingüística en los casos de sindrome de Asperger ha sido imprescindible dar cabida a vertientes de praxis que subrayen las dificultades detectadas en la toma de turnos, en el lenguaje indirecto, en la participación en un marco conversacional.

${ }^{6}$ La Gramática de construcciones descansa en el principio operativo de que los moldes, subsistemas y unidades emergen y se construyen paulatinamente a partir del estudio de los usos. No hay reglas a priori, sino que se observan a través de los datos (véanse, entre otros, Goldberg, 2006; Barlow y Kemmer, 1999 y Hoffmann y Trousdale, 2013). 


\subsection{Los corpus de habla disfuncional. Bases de elaboración y codificación de propiedades verbales}

De manera regular y casi natural, los repertorios de habla disfuncional se han confeccionado desde prismas pragmáticos. La presencia de eficacia comunicativa ha de ser criterio capital para dar relevancia a las producciones compiladas. De modo que todos los corpus de lingüística clínica, se encuentren o no etiquetados, contienen indicadores pragmáticos importantes. Primero, porque todos ellos se hallan definidos en cuanto a coordenadas comunicativas y a datos relativos a los participantes y a las actividades realizadas. Y segundo, porque en buena parte de los inventarios las muestras han sido promovidas o elicitadas siguiendo pautas previas de hormas y de protocolos pragmáticos. Por tratarse de corpus especializados y en esferas en que se necesita documentación por no disponer de fuentes de referencia, los repertorios de lenguaje disfuncional proceden de principios de compilación y sistematización definidos. Hay plantillas de registros de datos en baterías y test de evaluación del lenguaje que orientan asimismo la elaboración de corpus y su organización. Precisamente, el grueso de sistemas para estimar el lenguaje hace descansar sus bases y, sobre todo, proyecta su puesta en práctica en la visión integral de las producciones verbales. De entrada, por el papel que se adjudica a los interlocutores y a los estímulos para la interacción, motivo por el que se dan instrucciones para elicitar respuestas o recoger datos ${ }^{7}$. Además, por la importancia atribuida al entorno como fuente impulsora de riqueza y variación verbal, hasta el punto de introducirlo como parámetro esencial de la dinámica comunicativa. En el protocolo The Pragmatics Profile of Early Communication Skills (Dewart y Summers, 1988: 17) se insiste en la idoneidad de promover las interacciones en distintos marcos y con objeto de lograr indicios sobre confort y soltura lingüística (cfr. Smith y Leinonen, 1992: 254 y ss.). Finalmente, por la función estelar que detentan el propósito comunicativo y las intenciones, lo que explica que las pruebas evaluativas combinen expresión y comprensión independientemente de que pondere una de las destrezas: en cualquier caso, el paciente es emisor que compone un mensaje porque previamente

7 El reconocido como interlocutor-clave desempeña una función central al ser promotor desencadenante de dinámicas conversacionales. Sistemas de recogida de muestras, como los inventarios MacArthur (Fenson et al., 1993) incluyen indicaciones explícitas para la recogida de emisiones verbales:

"Por favor, marque la opción que más se parece a cómo su hijo empezó o está empezando a hablar"

"No se deben marcar las palabras que el niño imita, sólo las que produce espontáneamente"

"Marque la palabra, aunque la pronuncie de manera diferente/infantil". 
comprende lo que, con intenciones, quiere comunicar. La reciprocidad entre las destrezas de producción y de comprensión es consustancial a la valoración de los usos verbales por razón de su efectividad comunicativa. De ahí que ciertas baterías, como es el caso del test de Miller y Paul (1995), atribuyan primacía a la comprensión del sentido pragmático -como antesala de la expresión de usos comunicativamente relevantes-, con insistencia en la necesidad de valorar la comprensión discursiva global antes que la comprensión léxica de palabras, así como la interpretación de significados no-literales y contenidos discursivos:

working out the literal meaning of what we read or hear-identifying the referents of single words and decoding the meaning relations within sentences- is just one part of the comprehension process [...] Other aspects of comprehension involve making judgments based on social, textual, scriptal, and other forms of prior knowledge to figure out what an utterance really means, in relation to what else has gone in the discourse and to the intentional state of the speaker. We will refer to this as the discourse level of comprehension. Both literal and discourse levels are crucial to a listener's ability to get the full range of meaning from language (Miller y Paul, 1995: 7).

En una línea similar, el protocolo de desarrollo pragmático de Dewart y Summers (1988) no es sino una guía de cómo proceder en el registro de elementos y características que muestren las habilidades comunicativas en dinámica evolutiva. Las secciones que se delinean para recoger datos sobre las muestras definen los cuatro indicadores básicos de funcionamiento regular de la interacción comunicativa. A saber, (1) intenciones comunicativas (sean conscientes y controladas, sean imitadas o sean automatizadas); (2) respuesta a la comunicación (sea respuesta lingüística o sea respuesta no-verbal); (3) interacción y conversación; y (4) variación verbal ligada a diferentes contextos. Estas dimensiones prevén la adecuación y la eficacia discursiva. Sólo los marcos comunicativos facilitan la consideración de patrones funcionales. De ahí la importancia de recabar emisiones naturales de habla y no limitar la obtención de datos a cuestionarios formales cerrados en sus respuestas y valoraciones ${ }^{8}$. Las baterías de estimación cualitativa y no estandarizada van de la mano de la elaboración de corpus de habla disfuncional: sus hormas de registro de datos son orientación para

${ }^{8}$ Los test estandarizados que definen la evaluación sobre respuestas a cuestionarios cerrados no se acomodan al habla genuina y natural de los pacientes, así que deben sustituirse o complementarse acudiendo a procedimientos cualitativos que permitan valorar el discurso. Las baterías y los test que sopesan el habla peculiar deben respetar las características singulares de los procesos y no pueden perder de vista su naturaleza evolutiva (Fernández Pérez, 2005, 2014). 
recogida de muestras, y, a su vez, los repertorios procedentes de recolectar producciones son marcos referenciales en los que beber para establecer escalas en tales baterías.

El depósito global de corpus clínicos más importante y reconocido es el que contienen el CHILDES, Child Language Data Exchange System (<http:// childes.talkbank.org $>$ ) y el TALK BANK (<https://talkbank.org/>) sitios creados y gestionados por el equipo de MacWhinney desde los años noventa, con un sistema de transcripción y codificación unificado que facilita la acumulación documental de lenguaje peculiar en dimensiones interlingüísticas y en procesos dinámicos de desarrollo (adquisición de la lengua) y de trastorno comunicativo. En el CHILDES se hallan más de 70 corpus clínicos de más de una docena de lenguas, con aportación importante en inventarios de disfunciones en edad infantil y con repertorios abundantes en un síndrome como la afasia ${ }^{9}$. Son cerca de 50 los corpus con muestras de trastornos en edad infantil, casi todos ellos relativos al inglés y en buena parte centrados en SLI (Specific Language Impairment) y en síndrome de Down, si bien hay también algunos repertorios del espectro autista y concretamente de síndrome de Asperger, así como repertorios con alteraciones fónicas y ubicados en Talk Bank (<https://talkbank.org/>). En lo que se refiere al español, son cinco corpus clínicos de disfunciones en edad infantil y tres repertorios de trastornos en edad adulta (Afasia, Alzheimer y síndrome de Asperger) los que figuran en el CHILDES:

- $\quad$ el de Oviedo (<http://childes.talkbank.org/access/Clinical/Ovie do.html >), con muestras de dos hablantes uno con síndrome de Williams y otro con SLI (entre 7 y 8 años);

- $\quad$ el corpus $M O C$ (<http://childes.talkbank.org/access/Clinical/ MOC.html>) que incluye datos de un niño sordo con implante coclear (entre $1 ; 6$ y $5 ; 5)$;

\footnotetext{
${ }^{9}$ Hay otros sistemas y sitios de registro y depósito de datos de habla peculiar. Entre ellos están:

- MoDyCo (Modèles, Dynamiques, Corpus, <http://www.modyco.fr/>), con soporte económico del gobierno galo, y bajo la coordinación de Edy Veneziano (Université Paris-Descartes),

- LENA (Language Environment Analysis, <http://www.lenafoundation.org/ProSystem/ Overview.aspx $>$ ), especialmente centrado en el espectro autista,

- HUMAN SPEECHOME PROJECT (<https://www.media.mit.edu/research/groups/ 1446/human-speechome-project $>$ ) Sistema audiovisual e informático avanzado para recoger y analizar materiales comunicativos de manera continuada,

- ELAN (<http://www.lat-mpi.eu/tools/elan/>) ELAN is a professional tool for the creation of complex annotations on video and audio resources. Se inscribe en el macroplan The Language Archiving Technology, diseñado en el Max Planck Institute for Psycholinguistics de Nimega, Holanda.
} 
- $\quad$ el corpus Granada (<http://childes.talkbank.org/access/Clinical/ Granada.html $>$ ), con muestras de dificultades fónicas (entre 3;1 y $5 ; 11)$ y que se ha incluido en PhonBank (<http://phonbank.talk bank.org/access/>);

- y la aportación de PERLA (<http://childes.talkbank.org/access/ Clinical/PERLA.html>) en la sección de síndrome de Down (dos participantes de 10 y 17 años).

En cuanto a los corpus de trastornos en edad adulta, figuran en el TALK BANK el corpus Aphasia PERLA (<http://aphasia.talkbank.org/access/ Spanish/NonProtocol/PerLA.html>), de sujetos afásicos (13 fluentes y 19 no fluentes), el repertorio Dementia PERLA (<https://talkbank.org/access/ DementiaBank/Spanish/PerLA.html $>$ ), que incluye 27 transcripciones de 21 pacientes de Alzheimer, y el corpus ASD PERLA-Muñoz (<https://talk bank.org/access/ASDBank/Spanish/PerLA.html>), en el apartado de trastornos ASD (Autism Spectrum Disorder), que contiene 20 transcripciones con muestras de Asperger.

Buena parte de estos inventarios definen las coordenadas comunicativas en que tienen lugar las interacciones, con aclaraciones propias de la perspectiva pragmática adoptada, a saber (1) las condiciones de observación y el entorno natural de los intercambios verbales, con especificaciones acerca de los medios técnicos de grabación de muestras y de la cadencia en su recogida; (2) los parámetros comunicativos y de interacción que se han subrayado como pertinentes, conciernan a los participantes (rasgos de identidad individual y social), a los modos discursivos (conversación, explicación, narración, diálogo), a las modalidades expresivas (rechazo, petición, pregunta) ligadas a actividades y que desencadenan actos de habla, y a las intenciones y propósitos; y (3) los procedimientos utilizados para abordar el análisis y las anotaciones codificadas de modo sistemático. Los detalles de etiquetado pragmático específicos en cada repertorio suelen refinarse en consonancia con las características comunes del trastorno. De modo que en corpus de niños con SLI (Specific Language Impairment), tal y como señala Conti-Ramsden en uno de sus repertorios con etiquetado MOR (Morfología) en CHILDES (<http://childes.talkbank.org/access/ClinicalMOR/Conti1.html >) (cfr. Conti-Ramsden y Friel-Patti, 1987), se anoten rasgos como los siguientes -significativos todos ellos para examinar las reducidas destrezas de participación en disfunciones SLI-:

- Conversaciones que, al menos, se ajusten a "Two or more turns linked together by a focus on a particular topic".

- Turnos, sean nuevos $(\$ N E W)$, continuativos $(\$ C O N)$, o finalicen (\$END), 
- Turnos continuativos, según sean imitación (IMI), refundición simple (SR), refundición compleja (CR), preguntas o demandas (IN), preguntas para clarificar (RC), respuestas (RES), interacciones (INT),

- Finalización de turno, sea natural (NE), sea por cambio de tema por parte de los padres (TCP), sea por cambio de tema por parte del niño (TCC), o sea cambio por otro motivo (TCO).

Se incluyen, además, algunas etiquetas relativas a los estilos de interacción, como:

- Inicio de conversación verbal (VI), o no inicio verbal (NVI),

- Respuesta verbal (VR), o no verbal (NVR),

- Autocontinuación verbal (SV), o ausencia de autocontinuación (SNV).

De manera similar, las anotaciones en producciones de, por ejemplo, el sindrome de Asperger incluyen códigos alusivos al lenguaje indirecto, a la toma de turnos y al índice de participación conversacional, o al lenguaje figurado.

En lo que concierne a los repertorios de afásicos, si bien resulta predominante el etiquetado del agramatismo ${ }^{10}$ como síndrome común del trastorno, también se incorporan anotaciones que subrayan las destrezas ejecutivas y comunicativas del sujeto. Hasta tal punto alcanza la importancia comunicativa valorada en términos de eficacia y efectividad interactiva en el lenguaje idiosincrático de las disfunciones verbales, que hay propuestas que se interrogan acerca de los requisitos del lenguaje para que comunique. Se acude a las propiedades de diseño (Hockett y Altmann, 1968) y sobre su presencia y pertinencia en las producciones de un paciente se comprueba los grados de comunicabilidad y la posible afección de la ejecución verbal en el sujeto $^{11}$. El objeto es detectar si en el manejo de la lengua el sujeto produce emisiones en las que se evidencie control y autoreflexividad metapragmática, lo que prueba el mantenimiento de la función ejecutiva (Rosell Clarí y Hernández Sacristán, 2014).

Resulta interesante destacar que en el conjunto de producciones compiladas en el caso de afásicos se incluyen siempre dimensiones narrativas y conversacionales. Desde los protocolos pioneros en su planteamiento in-

${ }^{10}$ El síndrome del agramatismo afecta de modo específico según la tipología de la lengua, su incidencia se hace visible en reducción de morfemas si se trata de lenguas flexivas y en esquemas peculiares de orden de palabras cuando la lengua tiene orden fijo (Menn y Obler, 1990).

11 Perkins (2000, 2005 y 2014) insiste en la importancia de tomar en cuenta las bases cognitivas que intervienen en la habilidad pragmática básica y en la habilidad pragmática compleja. Entre esas dimensiones cognitivas están: la función ejecutiva, la memoria, el afecto, la habilidad inferencial, la cognición social, la teoría de la mente y el conocimiento del mundo. 
tegral de la lengua y por el enfoque interlingüístico adoptado, como es el definido en Menn y Obler (1990) -centrado en el agramatismo y que no obstante incluye dichas vertientes conversacionales y narrativas, tal y como se avanza en el subtítulo: A Cross-Language Narrative Sourcebook-, o el enfoque pragmático de Prutting y Kirchner (1983), hasta los más actuales como el Test Barcelona -ahora integrado, y que contiene una dimensión para afásicos (<http://www.test-barcelona.com/es/test-barcelona/programaintegrado/122-ficha-tecnica.html >)-, o los protocolos recomendados en el CHILDES para abordar las producciones de afásicos $(<\mathrm{http}: / /$ aphasia.talk bank.org/access/Protocol.html>), en todos ellos se hacen figurar parámetros pragmáticos que faciliten la descripción y el valor de los recursos comunicativos de los pacientes. Así, el corpus PERLA con muestras de pacientes afásicos en español (Fernández-Urquiza y Gallardo-Paúls, 2015) se ha anotado en características de pragmática enunciativa (actos de habla), textual (turnos) e interactiva (proyecciones), y con el cometido -tal y como se señala en la presentación del inventario (<http://aphasia.talkbank.org/ access/Spanish/NonProtocol/PerLA.html>) en Aphasia TALK BANK- de

assessing the pragmatic efficacy and the communicative success of aphasic speakers during spontaneous conversation.

Hay un factor capital que conviene tener presente en Lingüística de corpus y siempre que se acometa el estudio del habla y la interacción. Se trata del dinamismo procesual asociado al uso natural del lenguaje y que debe notarse en las compilaciones verbales. En la esfera de los trastornos comunicativos de ningún modo puede obviarse la perspectiva evolutiva: las variaciones y los cambios son señales que indican procesos de avance, retroceso o estancamiento de competencias en el manejo del lenguaje y en el éxito de la interacción. Las escalas de progreso y los percentiles que los test de evaluación del lenguaje incluyen reflejan las variaciones evolutivas en distintos niveles. La recomendación es someterse a evaluación cada cierto tiempo con objeto de comprobar el dinamismo y descartar el anquilosamiento. Algunas baterías incluso establecen niveles funcionales correlacionados entre componentes de la lengua. Un ejemplo es el BLADES (Bristol Language Development Scales, Gutfreund et al., 1989). En el BLADES se manejan criterios básicos relevantes para distribuir la evolución pareja en las dimensiones pragmática, semántica y sintáctica. A saber, funciones comunicativas (ostensión, llamada, petición directa e indirecta, explicación...) en la vertiente pragmática, puestas en paralelo con significados proposicionales (locativo, agentivo, estado...), con temporalidad/aspectualidad (pasado, futuro, habitual...), modalidad (posibilidad, obligación...) y contenidos frasales (nombre, adjetivo, pronombre...), en la semántica; y con conjunciones, estructuras frasales y construcciones oracionales, en la sintaxis. El enfoque glo- 
bal del seguimiento conjunto requiere del prisma comunicativo que confiere valor a las producciones aun siendo peculiares. Únicamente desde este planteamiento integral es posible la valoración de la competencia comunicativa del sujeto.

Los repertorios con datos verbales procedentes de trastornos comunicativos han de ser corpus de seguimiento (longitudinales) o comparativos (interseccionales) que permitan reflejar el dinamismo y contrastar los cambios. De algún modo, la tarea investigadora en la esfera de corpus-driven es continua, la construcción de inventarios enriquece la documentación de cualquier trastorno. Asimismo, las indagaciones corpus-oriented deben conducir a anotaciones cada vez más precisas, incrementar el etiquetado significa haber observado la actividad verbal permanente y constata la necesidad y la importancia de ir detallando rasgos singulares en las producciones compiladas. El análisis del discurso en las muestras y según los propios rasgos expresivos, como son las repeticiones, los turnos de intervención, las reparaciones, los elementos de cohesión e interacción, las partículas de enlace, los hilos discursivos, o cualesquiera otros indicadores de tácticas conversacionales y comunicativas, aquilata el seguimiento evolutivo y destaca los aspectos pertinentes en cada trastorno. No en vano la variación en la longitud del turno es significativa en afasias, como también es capital el seguimiento del número de turnos en niños SLI (Specific Language Impairment), o como son centrales los hilos nuevos de conversación en casos de Asperger.

Los corpus de Lingüística clínica no sólo tienen significado por ser resultado de compilación y sistematización de producciones peculiares, sino que además están llamados a ser fuente en la que beber para disponer de marco de referencia y comparar casos particulares. La valoración requiere comparar dentro del grupo. Los corpus de Lingüística clínica definen lo que es propio en cada trastorno y determinan en qué rasgos y aspectos, aunque sean idiosincráticos, radica la efectividad comunicativa. Facilitan el hallazgo del código en el puzle que mencionaba Crystal.

\section{RECAPITULACIÓN Y CONCLUSIONES}

Pragmática y Lingüística de corpus focalizan su materia de interés en los usos verbales. La ontología real y factual del habla se aborda de modo particular en cada una de las áreas: para hallar ocurrencias y colocaciones en el caso de la Lingüística de corpus, y para sistematizar principios y reglas en los intercambios comunicativos si se trata de Pragmática. En cualquier caso, y desde una visión integral de la lengua que no prime componentes, moverse en Lingüística de corpus requiere prismas pragmáticos, y asimismo, la esfera pragmática se cultiva únicamente si se dispone de repertorios de datos. 
La intersección entre ambos campos -consecuencia de sus pretensiones en una misma materia, la parole- se hace patente tanto en tareas de confección de corpus (corpus-driven), como en cometidos de anotación en inventarios (corpus-oriented, corpus pragmatics). La presencia de perspectiva pragmática en la elaboración de fuentes de datos es indisociable de la definición y del tratamiento de los materiales: la recolección de muestras, los parámetros para organizarlas en el depósito, las variables generales para determinar la sistemática de datos, son indicadores imprescindibles en el proceso. Por su parte, los pormenores descriptivos de praxis se visibilizan en labores de etiquetado de los muestrarios (corpus pragmatics) cuando se trata de anotar características relevantes sobre las que descansan aspectos pertinentes de eficacia comunicativa y que sostienen las reglas de uso (esperable o peculiar).

Los fundamentos pragmáticos de los corpus de lenguaje disfuncional resultan indispensables porque sobre ellos se asienta el criterio para decidir la relevancia comunicativa de las producciones idiosincráticas: la efectividad es la clave. El habla disfuncional interesa porque comunica a pesar de sus singularidades, está cargada de eficacia porque disfruta de valor de interacción. De modo que el peso comunicativo del habla disfuncional radica en su fuerza de intercambio, el papel estelar de la perspectiva pragmática es incontestable en Lingüística clínica.

Los repertorios de lenguaje disfuncional son, por definición, fuentes de datos pragmáticos. Contengan o no anotaciones. La investigación sobre muestras de lenguaje mermado tiene el objetivo de caracterizar el código peculiar propio del trastorno. No cabe obviar, por tanto, ni la presencia de tácticas verbales especiales, ni tampoco las estrategias no verbales utilizadas, así como aquellos factores de contexto, motivación, temas, interlocutores, que puedan activar o repercutir el potencial comunicativo del paciente. El papel del interlocutor (o interlocutores) se vuelve asimismo fundamental para otorgar el valor debido a las producciones por su eficacia: el marco dialógico se vuelve clave en los corpus de Lingüística clínica.

El etiquetado pragmático en los inventarios de datos verbales clínicos destaca las características propias del déficit. Los rasgos que definen el sistema comunicativo del trastorno o del paciente constituyen el objetivo primordial en tareas de corpus pragmatics en Lingüística clínica. El análisis del discurso en las muestras y según los propios rasgos expresivos, como son repeticiones, turnos de intervención, reparaciones, elementos de cohesión e interacción, partículas de enlace, hilos discursivos, u otros indicadores de tácticas conversacionales y comunicativas, forma parte del seguimiento evolutivo y acentúa los aspectos pertinentes en cada trastorno. Así, se han anotado, entre otros, la variación en la longitud del turno, significativa en afasias; el número de turnos, capital en niños SLI (Specific Language Impairment); o los hilos nuevos de conversación, centrales en casos de Asperger. 


\section{BIBLIOGRAFÍA}

Ajjmer, K. (2002): English Discourse Particles: Evidence from a Corpus, Amsterdam/ Philadelphia, John Benjamins.

Ajjmer, K. (2015): "Pragmatic markers", en K. Aijmer y Ch. Rühlemann (eds.), Corpus Pragmatics. A Handbook, Cambridge, Cambridge University Press, págs. 195-218.

Ajjmer, K. y Rühlemann, Ch. (eds.) (2015): Corpus Pragmatics. A Handbook, Cambridge, Cambridge University Press.

Allen, J. y CoRe, M. (1997): Draft of DAMSL: Dialog Act Markup in Several Layers (en línea: <https://www.cs.rochester.edu/research/speech/damsl/Revised Manual/>). Consulta 15/06/2017.

Andersen, G. (2001): Pragmatic Markers and Sociolinguistic Variation: A Relevance Theoretic Approach to the Language of Adolescents, Amsterdam/Philadelphia, John Benjamins.

Barlow, M. y Kemmer, S. (1989): Usage Based Models of Language, Chicago, The University of Chicago Press.

Battaner, E. (2014): Las ideas lingüisticas de John R. Firth, Münster, Nodus Pub.

Berber Sardinha, T. (2004): Lingüistica de corpus, Brasil, Editora Manole.

Biber, D., Johansson, S., Leech, G., Conrad, S. y Finegan, E. (1999): The Longman Grammar of Spoken and Written English, London, Longman.

BIBER, D. (2006): University language: A corpus-based study of spoken and written registers, Amsterdam/Philadelphia, John Benjamins.

BIBER, D. (2010): "Corpus-based and corpus-driven analyses of language variation and use", en B. Heine y H. Narrog (eds.), The Oxford Handbook of Linguistic Analysis, Oxford, Oxford University Press, págs. 159-192.

BiBER, D. y GRAY, B. (2011): "Corpus approaches to the study of discourse", en K. Hyland y B. Paltridge (eds.), The Continuum Companion to Discourse Analysis, London, Continuum, págs. 138-154.

Bublitz, W. y NorRICK, N. (2011): "Introduction: The burgeoning field of pragmatics”, en W. Bublitz y N. Norrick (eds.), Foundations of Pragmatics, Berlin, Mouton de Gruyter, págs. 1-20.

Chafe, W. (1992): "The importance of corpus linguistics to understanding the nature of language", en J. Svartvik (ed.), Directions in Corpus Linguistics. Proceedings of Nobel Symposium 82, Berlin, Mouton de Gruyter, págs. 79-97.

Conti-Ramsden, G. y Friel-Patti, S. (1987): "Mothers' discourse adjustments to language impaired and non-language-impaired children”, Journal of Speech and Hearing Disorders, 48, págs. 360-367.

Crystal, D. (1980): Introduction to Language Pathology, London, Edward Arnold. Traducción al español de M. L. Sánchez Bernardos, Patología del lenguaje, Madrid, Cátedra, 1983. 
Danescu-Niculesco-Mizil, D., Sudhof, M., Jurafsky, D., Leskovec, J. y Potts, C. (2013): A computational Approach to politeness with application to social factors. Proceedings of ACL 2013 (en línea: <https://web.stanford.edu/ jurafsky/pubs/politeness-acl13.pdf $>$ ). Consulta 15/06/2017.

Dewart, H. y Summers, S. (1988): The Pragmatics Profile of Early Communication Skills, Windsor, NFER-Nelson.

Felder, E., MÜller, M. y Vogel, F. (eds.) (2011): Korpuspragmatik: Thematische Korpora als Basis diskurslinguistischer Analysen, Berlin, W. de Gruyter.

Fenson, L., Dale, P., Reznick, S., Thal, D., Bates, E., Hartung, J., Pethick, S. y ReILly, J. (1993): The MacArthur Communicative Development Inventories, San Diego, CA, Singular.

Fernández López, I. y Fernández Pérez, M. (2014): "Sistemas de valoración del habla infantil”, en M. Fernández Pérez (coord.), Lingüistica y déficit comunicativos, Madrid, Síntesis, págs. 101-139.

Fernández López, I. y Prego VÁzQuez, G. (2014): "Exploración lingüística del habla infantil”, en M. Fernández Pérez (coord.), Lingüistica y déficit comunicativos, Madrid, Síntesis, págs. 45-99.

FERnÁndez Pérez, M. (2005): “¿Cómo evaluar el lenguaje infantil?”, en E. Serra y M. Veyrat (eds.), Estudios de Lingüistica Clínica/4. Problemas de eficacia comunicativa. Descripción, detección, rehabilitación, Valencia, Universitat de València, págs. 55-78.

FERNÁNDEZ PÉREZ, M. (2014): "Lingüística y déficit comunicativo. Lingüística clínica y Logopedia”, en M. Fernández Pérez (coord.), Lingüistica y déficit comunicativos, Madrid, Síntesis, págs. 19-44.

FERNÁNDEZ PÉREZ, M. (coord.) (2014): Lingüistica y déficit comunicativos. ¿Cómo abordar las disfunciones verbales?, Madrid, Síntesis.

Fernández-Urquiza, M. y Gallardo-Paúls, B. (2015): “Adaptación del formato CHAT para la transcripción y codificación de los datos de lenguaje patológico del corpus PERLA”, Oralia, 18, págs. 81-102.

Goldberg, A. (2006): Constructions at Work. The Nature of Generalization in Language, Oxford, Oxford University Press.

Gilquin, G. y GRIES, S. (2009): "Corpora and experimental methods: a state ofthe-art review”, Corpus Linguistics and Linguistic Theory, 5 (1), págs. 1-26.

Gutfreund, M., Harrison, M. y Wells, G. (1989): BLADES, Bristol Language Development Scales, London, NF-Nelson.

Gray, B. y Biber, D. (2015): "Stance markers", en K. Aijmer y Ch. Rühlemann (eds.), Corpus Pragmatics. A Handbook, Cambridge, Cambridge University Press, págs. 219-248.

Gries, S. (2010): "Methodological skills in corpus linguistics: A polemic and some pointers towards quantitative methods”, en T. Harris y M. Moreno Jaén (eds.), Corpus Linguistics in Language Teaching, Frankfurt, Peter Lang, págs. 121-146.

HALlidAY, M. A. K. (1992): "Language as system, language as instance: the corpus as a theoretical construct", en J. Svartvik (ed.), Directions in Corpus Linguistics. Proceedings of Nobel Symposium 82, Berlin, Mouton de Gruyter, págs. 61-78. 
Hockett, Ch. F. y Altmann, R. (1958): “A note on design features”, en Th. Sebeok (ed.), Animal communication: techniques of study and results of research, Bloomington, IULC, págs. 61-72.

Hoffmann, Th. y Trousdale, G. (eds.) (2013): The Oxford Handbook of Construction Grammar, Oxford, Oxford University Press.

Hunston, S. (2002): Corpora in Applied Linguistics, Cambridge, Cambridge University Press.

Hunston, S. (2008): "Collection strategies and design decisions", en A. Lüdeling y M. Kytö (eds.), Corpus linguistics: An international handbook, Berlin, Walter de Gruyter, págs. 154-168.

Hunston, S., Charles, M. y Pecorari, D. (eds.) (2009): Academic Writing: at the interface of corpus and discourse, London, Continuum.

Hunston, S. (2010): "How can a corpus be used to explore patterns?", en A. O'Keeffe y M. McCarthy (eds.), Routledge Handbook of Corpus Linguistics, London, Routledge, págs. 152-166.

Jakobson, R. (1956): "Deux aspects du langage et deux types d'aphasies", Essais de Linguistique Générale (traduit par N. Ruwet de l'original, Fundamentals of Language, The Hague), Paris, éditions de Minuit, 1963, págs. 43-67.

Jørgensen, A. M. y Eguía, E. (2014): "Presentación de COLA, un corpus oral de lenguaje adolescente en línea”, en S. A. Eiríksdóttir (ed.), Actes du XIXème Congrès des romanistes scandinaves. Du 12 au 15 août 2014, Université d'Islande, accesible en <http://conference.hi.is/rom14/files/2015/08/ANETTEJ\%C3 \%96RGENSEN-PADILLA.pdf $>$ ). Consulta 15/06/2017.

Jucker, A. H. y TAavitsainen, I. (2014): "Diachronic corpus pragmatics: Intersections and interactions", en I. Taavitsainen, A. H. Jucker y J. Tuominen (eds.), Diachronic Corpus Pragmatics, Amsterdam/Philadelphia, John Benjamins, págs. 3-26.

Kallen, J. L. y Kirk, J. (2012): SPICE-Ireland: A User's Guide, Belfast, Cló Ollscoil na Banríona.

MacWhinney, B. (2000): The CHILDES Project: Tools for Analyzing Talk. Volume 1: Transcription format and programs. Volume 2: The Database, Mahwah, NJ, Lawrence Erlbaum Associates.

Mactear, M. y Conti-Ramsdem, G. (1989): “Assessment of Pragmatics”, en K. Grundy (ed.), Linguistics in Clinical Practice, London, Taylor y Francis, págs. 150-167.

Menn. L. y Obler, L. (1990): Agrammatic Aphasia. A Cross-Language Narrative Sourcebook, Amsterdam, John Benjamins.

Miller, J. y PAul, R. (1995): The Clinical Assessment of Language Comprehension, Baltimore, Brookes Publishing.

OCHS, E. (1996): "Linguistic resources for socializing humanity", en J. J. Gumperz y S. C. Levinson (eds.), Rethinking Linguistic Relativity, Cambridge, Cambridge University Press, págs. 407-437.

Perkins, M. R. (2000): “The Scope of Pragmatic Disability”, en N. Müller (ed.), Pragmatics in Speech and Language Pathology. Studies in Clinical Applications, Amsterdam/Philadelphia, John Benjamins, págs. 7-28. 
PERKINS, M. R. (2005): "Pragmatic ability and disability as emergent phenomena", Clinical Linguistics and Phonetics, 19 (5), págs. 367-377.

Perkins, M. R. (2014): "Pragmatics as interaction", en M. Ball, N. Müller y R. L. Nelson (eds.), Handbook of Qualitative Research in Communication Disorders, New York/London, Psychology Press Taylor, págs. 131-147.

Prutting, C. y Kirchner, D. (1983): “Applied Pragmatics”, en T. Gallagher y C. Prutting (eds.), Pragmatic assessment and intervention issues in language, New York, Academic Press, págs. 29-68.

Romero-Trillo, J. (2008): "Introduction: Pragmatics and corpus linguistics -a mutualistic entente", en J. Romero-Trillo (ed.), Pragmatics and corpus Linguistics, Berlin, Mouton de Gruyter, págs. 1-10.

Romero-Trillo, J. (2014): Yearbook of Corpus Linguistics and Pragmatics. New Empirical and Theoretical Paradigms, Dordrecht, Springer.

Romero-Trillo, J. (2015): Yearbook of Corpus Linguistics and Pragmatics. Current Approaches to Discourse and Translation Studies, Dordrecht, Springer.

Rosell Clarí, V. y Hernández Sacristán, C. (2014): MetAphAs. Protocolo de exploración de habilidades metalingüisticas naturales en la afasia, Valencia, Nau Llibres.

Rühlemann, C. y O'Donnell, M. B. (2012): "Introducing a corpus of conversational narratives: Construction and annotation of the Narrative Corpus", Corpus Linguistics and Linguistic Theory, 8 (2), págs. 313-350.

RÜHLEMAnN, Ch. y AjJMer, K. (2015): "Corpus pragmatics: laying the foundations", en K. Aijmer y Ch. Rühlemann (eds.), Corpus Pragmatics. A Handbook, Cambridge, Cambridge University Press, págs. 1-26.

Simpson, R., Briggs, S., Ovens, J. y Swales, J. (2002): The Michigan Corpus of Academic Spoken English, Ann Arbor, The Regents of the University of Michigan.

Smith, B. R. y Leinonen, E. (1992): Clinical Pragmatics. Unravelling the complexities of communicative failure, London/New York, Chapman y Hall.

Stent, A. (2000): The Monroe Corpus, The University of Rochester, accesible en línea: <https://www.cs.rochester.edu/research/speech/monroe/>. Consulta $15 / 06 / 2017$.

Svartvik, J (ed.) (1992): Directions in Corpus Linguistics. Proceedings of Nobel Symposium 82, Berlin, Mouton de Gruyter.

TEubert, W. (2005): "My version of corpus linguistics", International Journal of Corpus Linguistics, 10(1), págs. 1-13.

Teubert, W. y Krishnamurthy, R. (2007): "General Introduction”, en W. Teubert y R. Krishnamurthy (eds.), Corpus Linguistics. Critical Concepts in Linguistics, London, Routledge, págs. 1-13.

Timmis, I. (2015): “Tails”, en K. Aijmer y Ch. Rühlemann (eds.), Corpus Pragmatics. A Handbook, Cambridge, Cambridge University Press, págs. 304-330.

Tognini-Bonelli, E. (2001): Corpus Linguistics at Work, Amsterdam/Philadelphia, John Benjamins.

Tognini-Bonelli, E. (2012): "The evolution of Corpus Linguistics", en A. O'Keeffe y M. McCarthy (eds.), The Routledge Handbook of Corpus Linguistics, London, Routledge, págs. 14-27.

Thompson, G. (2009): "Linguists would argue - biochemists conclude: A crossdisciplinary comparison of discourse act labeling in research articles", en S. 
Slembrouck, M. Taverniers y M. Van Herreweghe (eds.), From will to well: Studies in linguistics offered to Anne-Marie Simon-Vandenbergen, Ghent, Academic Press, págs. 405-414.

Thompson, G. y Hunston, S. (2006): "System and corpus: two traditions with a common ground", en G. Thompson y S. Hunston (eds.), System and Corpus. Exploring the Connections, London, Equinox, págs. 1-14.

Weisser, M. (2010): Annotating Dialogue Corpora Semi-Automatically: A Corpus-Based Approach to Pragmatics, Bayreuth, University of Bayreuth.

WeIsser, M. (2014): The Dialogue Annotation and Research Tool (DART), accesible en: <http://martinweisser.org/DART_scheme.html>. Consulta 15/06/2017.

Weisser, M. (2015): "Speech act and annotation”, en K. Aijmer y Ch. Rühlemann (eds.), Corpus Pragmatics. A Handbook, Cambridge, Cambridge University Press, págs. 84-112. 